

\section{Finding space}

"Depending on the application, footprint can be an important consideration," says Chang. To illustrate her point, Chang notes that for in vitro fertilization applications, the microscopes being used require sterile environments. This often means that a whole microscope needs to be contained within the very small confines of a tissue culture hood.

To deal with this issue and provide users with the maximum workspace possible within sterile enclosures, Nikon developed an inverted microscope called the Eclipse Ts2R, which is intermediate in size between a routine tissue culture microscope and a full research-grade microscope. With the design modifications, the Ts2R can fit easily within a laminar flow hood, enabling applications that require imaging in a sterile environment.

It's important to note that reducing the size of a microscope is not just a matter of making smaller components. As it turns out, there are fundamental challenges rooted in optical physics that need to be overcome. In a microscope, there is a minimum length in the optical path that must be maintained for proper imaging of samples. Chang says that while it is possible to use prisms, mirrors, and relay lenses to bend optical paths to fit into smaller instrument configurations, the additional accessory optics can also attenuate light transmission and therefore impact image quality. This illustrates one of the problems developers encounter when designing smaller instruments-the trade-off between size and capability. For this reason, some have turned to entirely new methodologies to reduce instrument size.

\section{Opening possibilities}

For decades, DNA sequencers have relied on imaging the incorporation of fluorescently tagged nucleotides into growing DNA strands in order to analyze base composition. This approach means that sequencing instruments have to possess sophisticated light detectors and high-speed cameras for data acquisition. To develop a smaller, less costly sequencing instrument, some developers have decided to follow a very different path.

Nanopore sequencing is an elegantly simple idea-likely the reason so many groups have worked on advancing this approach over the years. Very long strands of DNA transit through extremely small openings (called nanopores). As each base goes through a pore, it creates a unique charge difference that can be measured across the pore opening. By reading these charge differences, sequences can be decoded quickly without the need for imaging, saving both cost and time and reducing the space needed for instrumentation.

While the possibilities of nanopore sequencing seemed endless from the start, constructing and implementing a nanopore-based sequencing platform proved to be a significant technical challenge. In early studies, the DNA often

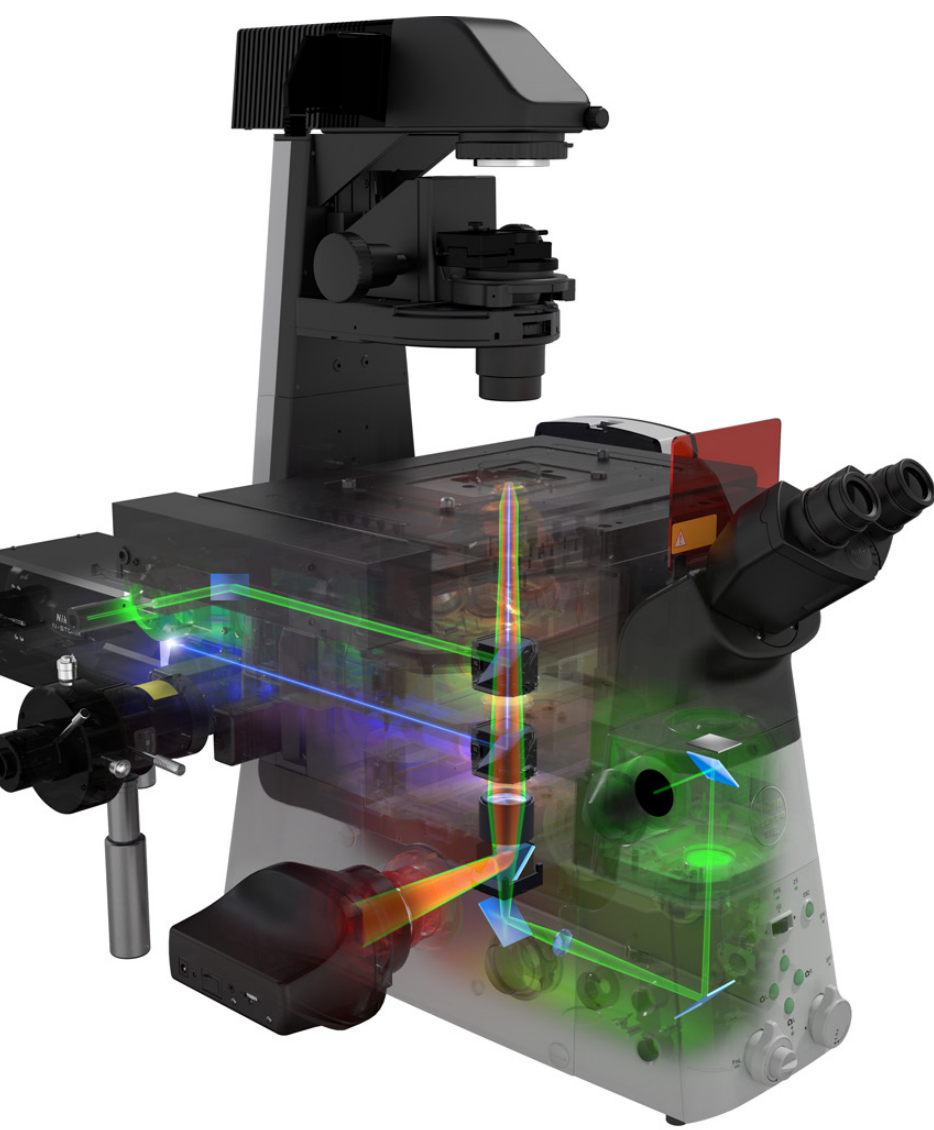

n illustration of the optical light path used in a light microscope. Credit: Nikon Instruments, Inc.

went through the opening too quickly to be read. Researchers reported minor successes as small sections of DNA were decoded in proof-of-concept experiments, supporting the technique's potential, but it seemed as if developers were still a long way from creating a robust nanopore sequencing system suitable for widespread use. That changed in early 2012.

In February of that year, the annual Advances in Genome Biology and Technology (AGBT) meeting was held at Marco Island in Florida. One of the most anticipated presentations was from a small UK-based company called Oxford Nanopore. The company's CEO, Clive Brown, intrigued the collection of sequencing enthusiasts assembled at the meeting by presenting the first data from a protein nanopore device, which the company intended to commercialize in the coming years. Perhaps more surprising than the data Brown showed, however, was the device itself-the entire sequencer was no bigger than a box of crayons and worked by simply plugging it into a laptop computer. At a time when the major sequencing platforms were the size of large filing cabinets, the idea of a sequencer that could fit snugly into your pocket stunned the scientists at the meeting, as well as the larger research community.

"Miniaturized sequencing in real-time would impact not only life science research but other industries as well," noted a company spokesperson from Oxford Nanopore. But to truly understand what this means, it is essential to look at Oxford Nanopore's first commercial nanopore system. 


\section{Road to handheld}

\section{sequencers}

Oxford Nanopore's flagship device, called the MinlON, is a full sequencer the size of computer thumb drive and weighing less than $100 \mathrm{~g}-\mathrm{a}$ stark contrast to the hundreds of pounds needed for most other sequencing systems. This contrast in footprint, providing the chance to have a truly mobile sequencing device, along with the initial sequencing data presented by Brown at the meeting, resulted in strong reactions ranging from hope to skepticism. But before the MinlON would officially be released, Oxford Nanopore needed to refine the technology.

The issue was that early MinION sequence output was low, and more troubling, accuracy was only approximately $75 \%$. In the world of sequencing, two metrics really matteroutput and accuracy- and neither can be compromised. The MinION prototype might have been small and portable, with far lower costs than any other sequencer on the planet, but none of that would matter if those two key metrics could not be improved.

To achieve the needed performance enhancements, Oxford developers focused on two key components: sensing across the nanopores and refining the enzyme used to assist the DNA in its transit through the protein pore. As electrical engineers worked to enhance sensing of electrical current, protein biologists engineered enzymes to increase the speed at which the enzyme fed the DNA into the pore. In the first iteration of the platform, DNA transited through the nanopore at a rate of 70 bases per second. With modifications, that rate has increased to 450 bases per second, with improved base calling.

Nanopore sequencing reached a major milestone this fall-researchers reported for the first time whole-genome sequencing of a human sample using Oxford Nanopore technology. Prior to this, whole-genome sequencing using the nanopore system had been reported for smaller, less complex microbial genomes. Multiple reports of mammalian genome sequencing open the technology to new applications.

While its smaller size is an obvious advantage, the MinlON's numbers are now turning heads too. According to Oxford Nanopore, a single flow cell can produce 5-10 gigabases of sequence data at a cost of $\$ 1000$. Accuracy has risen into the mid-90\% range for singleread runs, and consensus accuracy when both strands are sequenced can reach as high as $99.96 \%$. Nanopore sequencing now seems to have finally found its stride. The technology was one of Science magazine's top 5 options for scientific breakthrough in 2016.

\section{Flowing with the changes}

"Small foot-sized cytometers are important, as the cytometers are moving into many individual $\mathrm{PI}$ labs and individual cell biology labs, instead of traditional core facilities," says Xiaobo Wang, Chief Technology Officer for ACEA Biosciences, Inc. Iocated in San Diego, California. Wang knows a little about this trend toward smaller, more personal instrumentsACEA recently launched their own benchtop flow cytometry system, called Novocyte.

While size can be a consideration in the design of a new instrument or refinement of an older model, Wang is quick to note that for some instruments, smaller footprints can indeed be a consequence of advances in specific parts. In the case of a cytometer, the optical platform that is used in sample illumination and fluorescent light collection has been large in the past, resulting a larger instrument. Optical platforms are crucial in flow cytometer design since the number of lasers and detectors used is directly linked to the number of colors the instrument can detect and, therefore, the number of cell parameters that can be examined. Many researchers think more parameters are generally better, which until recently meant deciding between a smaller footprint instrument with less color detection capabilities and a larger machine with many lasers. But advances in laser technology in recent years have blurred these lines. According to Wang, current solid-state lasers are much smaller, yet they still provide a good-quality instrument. The same goes for photo-multiplier tubes and other light detectors, enabling multiple-color detection in a smaller instrument package.

"These new cytometers employ the latest optical components, including lasers, photodetectors, and electronic processing chips, and this has important consequences for instrument size and quality," says Wang, who helped develop the Novocyte system, 
which can detect up to 15 parameters with up to 3 lasers. There are still some parts of a flow cytometry system, however, where a tug-of-war between size and efficiency exists.

For flow cytometry to work, cells need to be driven into a single-file line for analysis. Most instruments accomplish this by placing the cells into the middle of a sheath fluid, which helps align them using hydrodynamic pressure. This pressure is generated using a sealed tube that can drive continuous sample delivery into the flow chamber. While this is happening, the sheath fluid is driven separately to surround and focus the sample stream in the flow cell.

According to Wang, instrument size will also depend in large measure on how this constant pressure on the sample is generated. For example, using a large external pressure generator pump means the sampling delivery sub-systems will be larger in size. Several modern small footprint cytometers instead utilize peristaltic pumps to drive the sample and the sheath fluid separately into the flow chambers, where the differential pressure between the sample and the sheath fluid can be maintained to keep a stable flow. For these instruments, Wang says, a small footprint is achieved naturally due to the small size of the pumps.

When it comes to the overall adoption of smaller footprint cytometers, although Wang does not know the exact numbers, he strongly suspects that uptake has been robust. This is likely due to the number of options now available to interested researchers. In addition to ACEA's Novocyte platform, several other benchtop

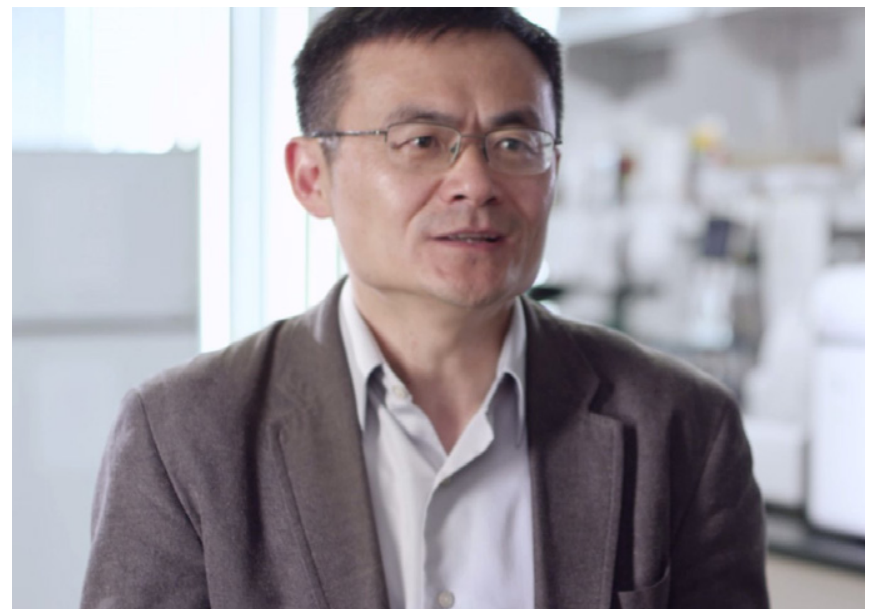

Xiaobo Wang from ACEA Biosciences says there is a growing adoption of smaller-footprint benchtop flow cytometers. Credit: ACEA Biosciences.

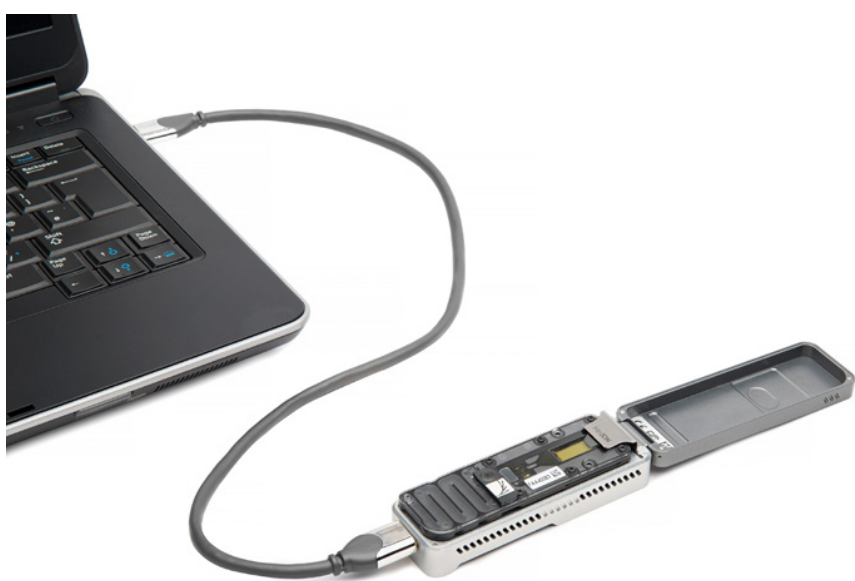

The MinION instrument from Oxford Nanopore Technologies is the size of a computer thumb drive, enabling users to sequence DNA samples at lab or field locations. Credit: Oxford Nanopore Technologies.

flow cytometers have emerged in recent years. EMD Millipore in Billerica, Massachusetts has developed the Guava easyCyte system, a microcapillary-based flow cytometer that differs from more traditional instruments by not needing a sheath fluid to drive cells (due to the microcapillary design), thereby resulting in its compact configuration. The CyFlow line of cytometers from Sysmex America, Inc. in Lincolnshire, Illinois, which looks to be the size of a small copier, presents another option for benchtop cytometry, with configurations for up to 13 parameters from 1 to 5 light sources. The end result of all this innovation in flow cytometry instrumentation is that researchers looking specifically for smaller instruments with different configurations and parameters now have more options for their labs.

Whether the move to smaller instrumentation is a conscious decision among developers, an outgrowth of advances in parts and basic electronics, or the result of a completely new methodology depends on the specific circumstances. But one thing is clear: Researchers now have the opportunity to reap the benefits of "tiny instruments" in their own labs and beyond, with some of these smaller instruments expanding the idea of the traditional lab setting.

According to Oxford Nanopore, new sequencing technology such as MinION creates new accessibility options, opening up applied areas where sequencing was not feasible before. Specifically, the company points to food and water testing as well as pest control as applications where access to rapid, lowcost sequencing data generated using a handheld instrument and a computer could have huge economic and health impacts.

Smaller instruments do create options for researchers. But more than just freeing up space, these latest advances in microscopy, DNA sequencing, and flow cytometry technology are providing powerful new ways to conduct research. As it turns out today's "tiny lab" could be just what life science needs.

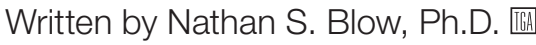

BioTechniques 62:8-12 January 2017

doi: 10.2144/000114490 